\title{
Singular Moser-Trudinger inequality with the exact growth condition on hyperbolic space
}

\section{Zhao Liu' and Lu Chen ${ }^{2 *}$}

\section{"Correspondence:}

luchen2015@mail.bnu.edu.cn

${ }^{2}$ School of Mathematical Sciences,

Beijing Normal University, Beijing,

100875, China

Full list of author information is

available at the end of the article

\begin{abstract}
In this paper, we are concerned with a singular version of the Moser-Trudinger inequality with the exact growth condition in the $n$-dimension hyperbolic space $\mathbb{H}^{n}$. Our result is a natural extension of the work of Lu and Tang in (J. Geom. Anal. 26:837-857, 2016).
\end{abstract}

MSC: $42 \mathrm{~B} 35 ; 42 \mathrm{~B} 37$

Keywords: exact growth; hyperbolic space; singular Moser-Trudinger inequality

\section{Introduction}

Let $W_{0}^{1, p}(\Omega)$ denote the usual Sobolev space, i.e., the completion of $C_{0}^{\infty}(\Omega)$ under the Sobolev norm

$$
\|u\|_{W_{0}^{1, p}(\Omega)}=\left(\int_{\Omega}|\nabla u|^{p}+|u|^{p} d x\right)^{\frac{1}{p}} .
$$

The classical Sobolev embedding theorem states $W_{0}^{1, n}(\Omega) \subset L^{q}(\Omega)$ for all $1 \leq q<\infty$ but $W_{0}^{1, n}(\Omega) \nsubseteq L^{\infty}(\Omega)$. One can check this by choosing the function $u(x)=\ln \left(\ln \frac{4 R}{\left|x-x_{0}\right|}\right)$ for some $R>0$, where $x_{0}$ is a fixed point in $\Omega$. It is natural to ask whether there exists an optimal embedding in this limiting case. Yudovic [2], Pohozaev [3] and Trudinger [4] showed that, for some $\alpha>0, W_{0}^{1, n}(\Omega)$ is continuously embedded into an Orlicz space with the Young function $\phi_{\alpha}(t)=\exp \left(\alpha|t|^{\frac{n}{n-1}}-1\right)$. Moser in [5] sharpened the exponent $\alpha$ and obtained the following result.

Theorem A ([5]) Let $\Omega$ be a bounded domain i $n \mathbb{R}^{n}(n \geq 2)$. Then there exists a positive constant $C_{n}$ and a sharp constant $\alpha_{n}=n \omega_{n-1}^{\frac{1}{n-1}}$ such that

$$
\frac{1}{|\Omega|} \int_{\Omega} \exp \left(\alpha|u|^{\frac{n}{n-1}}\right) d x \leq C_{n}
$$

for any $\alpha \leq \alpha_{n}$ and $u \in C_{0}^{\infty}(\Omega)$ with $\int_{\Omega}|\nabla u|^{n} d x \leq 1$, where $\omega_{n-1}$ is the area of the surface of the unit ball.

(c) The Author(s) 2017. This article is distributed under the terms of the Creative Commons Attribution 4.0 International License (http://creativecommons.org/licenses/by/4.0/), which permits unrestricted use, distribution, and reproduction in any medium, provided you give appropriate credit to the original author(s) and the source, provide a link to the Creative Commons license, and indicate if changes were made. 
The above inequality is often referred as the Moser-Trudinger inequality. Carleson and Chang [6] employed a symmetrization and rearrangement arguments to obtain the extremals of the Moser-Trudinger inequality when $\Omega$ is a disk in $\mathbb{R}^{2}$. Later, the results of Carleson and Chang were extended by Flucher [7] to arbitrary domains in $\mathbb{R}^{2}$, and by Lin [8] for arbitrary domains in $\mathbb{R}^{n}$. The existence of extremals of the Moser-Trudinger inequality was also extended to compact Riemannian manifold cases by $\mathrm{Li}$ in $[9,10]$. Cohn and $\mathrm{Lu}$ [11] were concerned with the sharp constants for the Moser-Trudinger inequality on a bounded domain on Heisenberg group $\mathbb{H}^{n}$. The singular version of the Moser-Trudinger inequality on the Heisenberg group was also proved by Lam, Lu and Tang in [12]. For more results as regards the Moser-Trudinger inequalities and applications in partial differential equations, please see [13-19] and the references therein.

A natural idea is to consider the Moser-Trudinger inequality in the whole space $\mathbb{R}^{n}$. Adachi and Tanaka in [20] proved the following nice result.

Theorem B ([20]) For $0<\alpha<\alpha_{n}$, there exists a positive constant $C_{n}$ such that

$$
\sup _{u \in W^{1, n}\left(\mathbb{R}^{n}\right), \int_{\mathbb{R}^{n}}|\nabla u|^{n} d x \leq 1} \int_{\mathbb{R}^{n}} \Phi\left(\alpha|u(x)|^{\frac{n}{n-1}}\right) d x \leq C_{n} \int_{\mathbb{R}^{n}}|u(x)|^{n} d x
$$

where $\Phi(t):=e^{t}-\sum_{i=0}^{n-2} \frac{t^{i}}{i !}$. Moreover, the constant $\alpha_{n}$ is sharp in the sense that if $\alpha \geq \alpha_{n}$, the supremum will become infinite.

They proved the sharpness of the exponent $\alpha$ by modifying a sequence of test functions introduced by Moser. In order to obtain the Moser-Trudinger inequality in the critical case $\alpha=\alpha_{n}$, Ruf [21] (in the dimension $n=2$ ) and Li and Ruf [22] (in the dimension $n \geq 3$ ) replaced the Dirichlet norm with the standard Sobolev norm, i.e.

$$
\|u\|_{W_{0}^{1, n}\left(\mathbb{R}^{n}\right)}=\left(\int_{\mathbb{R}^{n}}|\nabla u|^{n}+|u|^{n} d x\right)^{\frac{1}{n}}
$$

and obtained the Moser-Trudinger inequality in the whole space $\mathbb{R}^{n}$ in the case of $\alpha=\alpha_{n}$. Masmoudi and Sani in their elegant papers [23, 24] kept the two conditions $\alpha=\alpha_{n}$ and $\int_{\mathbb{R}^{n}}|\nabla u|^{n} d x \leq 1$. They proved the following.

Theorem C ([24]) For $n \geq 2$, there exists a positive constant $C_{n}$ such that

$$
\int_{\mathbb{R}^{n}} \frac{\Phi\left(\alpha_{n}|u|^{\frac{n}{n-1}}\right)}{(1+|u|)^{\frac{n}{n-1}}} d x \leq C_{n} \int_{\mathbb{R}^{n}}|u(x)|^{n} d x, \quad \forall u \in W^{1, n}\left(\mathbb{R}^{n}\right) \text { with } \int_{\mathbb{R}^{n}}|\nabla u|^{n} d x \leq 1
$$

Moreover, this inequality fails if the power $\frac{n}{n-1}$ in the denominator is replaced by any $p<\frac{n}{n-1}$.

The above result was also extended by Chen and Liu [25] to singular version through the change of variables developed by Dong and Lu in [17].

This paper is concerned with a singular version of Moser-Trudinger inequality with the exact growth condition on hyperbolic space $\mathbb{H}^{n}$. The hyperbolic space $\mathbb{H}^{n}(n \geq 2)$ is a complete and simply connected Riemannian manifold and has constant curvature equal to -1 . There exist many types of models for hyperbolic space $\mathbb{H}^{n}$. However, the most important 
models are the half-space model, the ball model, and the hyperboloid (or Lorentz) model. Throughout this paper, we are concerned about the ball model because we can use symmetry and rearrangement argument in this setting.

We define $B^{n}=\left\{x \in \mathbb{R}^{n}:|x|<1\right\}$ as a pen unit ball in $\mathbb{R}^{n}$ equipped with the Riemannian metric $g_{i j}=\left(\frac{1}{1-|x|^{2}}\right)^{2} \delta_{i j}$, which is referred to as the ball model of the hyperbolic space $\mathbb{H}^{n}$. A direct computation shows that the volume element of hyperbolic space $\mathbb{H}^{n}$ is given by $d V=\left(\frac{2}{1-|x|^{2}}\right)^{n} d x$ and $d(0, x)=\ln \frac{1+|x|}{1-|x|}$, where $d x$ denotes the Lebesgue measure in $\mathbb{R}^{n}$ and $d(0, x)$ denotes the hyperbolic distance between the origin and $x$. It is well known that the hyperbolic gradient $\nabla_{g}$ is defined as the $\nabla_{g}=\left(\frac{1-|x|^{2}}{2}\right)^{2} \nabla$, where $\nabla$ denotes the general gradient in $\mathbb{R}^{n}$.

Let $\Omega$ be a domain with finite measure on hyperbolic space $\mathbb{H}^{n}$. Denote $\|f\|_{L^{n}(\Omega)}=$ $\left(\int_{\Omega}|f|^{n} d V\right)^{\frac{1}{n}}$. A straightforward calculation yields

$$
\left\|\nabla_{g} f\right\|_{L^{n}(\Omega)}=\left(\int_{\Omega}\left\langle\nabla_{g} f, \nabla_{g} f\right\rangle_{g}^{n / 2} d V\right)^{\frac{1}{n}}=\left(\int_{\Omega}|\nabla f|^{n} d V\right)^{\frac{1}{n}}
$$

and

$$
\left\|\nabla_{g} f\right\|_{L^{n}\left(\mathbb{H}^{n}\right)}=\left(\int_{\mathbb{H}^{n}}\left\langle\nabla_{g} f, \nabla_{g} f\right\rangle_{g}^{n / 2} d V\right)^{\frac{1}{n}}=\left(\int_{B^{n}}|\nabla f|^{n} d V\right)^{\frac{1}{n}}
$$

We also define $W^{1, n}\left(\mathbb{H}^{n}\right)$ as the completion of $C_{0}^{\infty}\left(\mathbb{H}^{n}\right)$ with the norm

$$
\|u\|_{W^{1, n}\left(\mathbb{H}^{n}\right)}=\left(\int_{\mathbb{H}^{n}}\left|\nabla_{g} u\right|^{n}+|u|^{n} d V\right)^{\frac{1}{n}}
$$

The Moser-Trudinger inequality on hyperbolic space was first established by Mancini and Sandeep [26], they proved the Moser-Trudiner inequality on conformal discs. Lu and Tang in [27] considered the subcritical Moser-Trudinger inequality on high dimensional hyperbolic space. They proved the following result.

Theorem D For any $u \in W^{1, n}\left(\mathbb{H}^{n}\right)$ satisfying $\int_{\mathbb{H}^{n}}\left|\nabla_{g} u\right|^{n} d V \leq 1$, there exists a positive constant $C_{n}$ such that

$$
\int_{\mathbb{H}^{n}} \frac{\Phi\left(\alpha\left(1-\frac{\beta}{n}\right)|u|^{\frac{n}{n-1}}\right)}{(d(0, x))^{\beta}} d V \leq C_{n} \int_{\mathbb{H}^{n}} \frac{|u(x)|^{n}}{(d(0, x))^{\beta}} d V
$$

for any $\alpha<\alpha_{n}$. Furthermore the constant $\alpha_{n}$ is sharp in the sense that the inequality does not hold if we replace the constant $\alpha$ with any $\alpha \geq \alpha_{n}$.

Recently, Lu and Tang in [1] considered the sharp Moser-Trudinger inequality with the exact growth condition on hyperbolic space, they proved the following results.

Theorem $\mathrm{E}$ For any $u \in W^{1, n}\left(\mathbb{H}^{n}\right)$ satisfying $\int_{\mathbb{H}^{n}}\left|\nabla_{g} u\right|^{n} d V \leq 1$, there exists a positive constant $C_{n}$ such that

$$
\int_{\mathbb{H}^{n}} \frac{\Phi\left(\alpha_{n}|u|^{\frac{n}{n-1}}\right)}{(1+u)^{\frac{n}{n-1}}} d V \leq C_{n} \int_{\mathbb{H}^{n}}|u(x)|^{n} d V
$$


Furthermore, the power $\frac{n}{n-1}$ is sharp in the sense if the power $\frac{n}{n-1}$ is replaced by any $p<\frac{n}{n-1}$, the (1) become infinite.

Motivated by the above results, we consider the singular version of the Moser-Trudinger inequality with the exact growth condition on hyperbolic space. We state our results as follows.

Theorem 1 For any radially decreasing function $u \in W^{1, n}\left(\mathbb{H}^{n}\right)$ satisfying $\int_{\mathbb{H}^{n}}\left|\nabla_{g} u\right|^{n} d V \leq$ 1 , there exists a positive constant $C_{n}$ independent of $u$ such that

$$
\int_{\mathbb{H}^{n}} \frac{\Phi\left(\alpha_{n}\left(1-\frac{\beta}{n}\right)|u|^{\frac{n}{n-1}}\right)}{(1+u)^{\frac{n}{n-1}}(d(0, x))^{\beta}} d V \leq C_{n} \int_{\mathbb{H}^{n}} \frac{|u(x)|^{n}}{(d(0, x))^{\beta}} d V
$$

We verify that the power $\frac{n}{n-1}$ is optimal.

Theorem 2 If the power $\frac{n}{n-1}$ is replaced by any $p<\frac{n}{n-1}$, there exists a sequence of functions $\left\{u_{k}\right\}$ such that $\int_{\mathbb{H}^{n}}\left|\nabla_{g} u_{k}\right|^{n} d V \leq 1$, but

$$
\int_{\mathbb{H}^{n}} \frac{\Phi\left(\alpha_{n}\left(1-\frac{\beta}{n}\right) \mid u_{k} k^{\frac{n}{n-1}}\right)}{\left(1+u_{k}\right)^{p}(d(0, x))^{\beta}} d V\left(\int_{\mathbb{H}^{n}} \frac{\left|u_{k}(x)\right|^{n}}{(d(0, x))^{\beta}} d V\right)^{-1} \rightarrow \infty .
$$

This paper is organized as follows. In Section 2, we give some important lemmas which will play key roles in the proof of Theorem 1. In Section 3, we establish a singular version of Moser-Trudinger inequality with the exact growth condition on hyperbolic space (Theorem 1). In Section 4, we give the proof of the sharpness of the singular Moser-Trudinger inequality with the exact growth condition in Theorem 1.

\section{Some important lemmas}

In this section, we give some key lemmas which play an important role in the proof of Theorem 1.

Lemma 3 (see $[1,23]$ ) Given any sequence $a=\left\{a_{k}\right\}_{k \geq 1}$, let $\|a\|_{1}=\sum_{k=0}^{+\infty}\left|a_{k}\right|,\|a\|_{n}=$ $\left(\sum_{k=0}^{+\infty}\left|a_{k}\right|^{n}\right)^{1 / n},\|a\|_{(e)}=\left(\sum_{k=0}^{+\infty}\left|a_{k}\right|^{n} e^{k\left(1-\frac{\beta}{n}\right)}\right)^{1 / n}$ and $\mu(h)=\inf \left\{\|a\|_{(e)}:\|a\|_{1}=h,\|a\|_{n} \leq 1\right\}$.

Then, for any $h>1$, we have

$$
\mu^{n}(h) \sim \frac{e^{\left(1-\frac{\beta}{n}\right) h^{\frac{n}{n-1}}}}{h^{\frac{n}{n-1}}} .
$$

With the help of Lemma 3, one can obtain the following lemma.

Lemma 4 There exists a constant $C$ such that for, any nonnegative decreasing function $u$ with $u(R)>K^{\frac{1}{n}}$ and $\omega_{n-1} \int_{R}^{\infty}\left|u^{\prime}\right|^{n} t^{n-1} d t \leq K$ for some $R, K>0$,

$$
\frac{e^{\alpha_{n}\left(1-\frac{\beta}{n}\right) K^{-\frac{1}{n-1}} u^{\frac{n}{n-1}(R)}}}{u^{\frac{n}{n-1}}(R)} R^{n-\beta} \leq C \frac{\int_{R}^{\infty} u^{n} t^{n-\beta-1} d t}{K^{\frac{n}{n-1}}} .
$$


Proof By scaling, it suffices to show that, for any nonnegative decreasing function $u$ satisfying $u(1)>1$ and $\omega_{n-1} \int_{1}^{\infty}\left|u^{\prime}\right|^{n} t^{n-1} d t \leq 1$,

$$
\frac{e^{\alpha_{n}\left(1-\frac{\beta}{n}\right) u^{\frac{n}{n-1}(1)}}}{u^{\frac{n}{n-1}(1)}} \lesssim \int_{1}^{\infty} u^{n} t^{n-\alpha-1} d t .
$$

Let $h_{k}=\alpha_{n}^{\frac{n-1}{n}} u\left(e^{k / n}\right), a_{k}=h_{k}-h_{k+1}$ and $a=\left\{a_{k}\right\}$. Then $a_{k} \geq 0$ and

$$
\sum_{k \geq 0}\left|a_{k}\right|=h_{0}=\alpha_{n}^{\frac{n-1}{n}} u(1)
$$

Since

$$
\begin{aligned}
h_{k}-h_{k+1} & =\alpha_{n}^{\frac{n-1}{n}}\left(u\left(e^{\frac{k}{n}}\right)-u\left(e^{\frac{k+1}{n}}\right)\right) \\
& =\alpha_{n}^{\frac{n-1}{n}} \int_{e^{\frac{k+1}{n}}}^{e^{\frac{k}{n}}} u^{\prime}(t) d t \\
& =\alpha_{n}^{\frac{n-1}{n}}\left(\int_{e^{\frac{k}{n}}}^{e^{\frac{k+1}{n}}}\left|u^{\prime}(t)\right|^{n} t^{n-1} d t\right)^{\frac{1}{n}}\left(\int_{e^{\frac{k}{n}}}^{e^{\frac{k+1}{n}}} \frac{1}{t} d t\right)^{\frac{n-1}{n}} \\
& =\left(\omega_{n-1} \int_{1}^{\infty}\left|u^{\prime}(t)\right|^{n} t^{n-1} d t\right)^{\frac{1}{n}},
\end{aligned}
$$

we have

$$
\begin{aligned}
\|a\|_{n} & =\left(\sum_{k \geq 0}\left|a_{k}\right|^{n}\right)^{1 / n} \\
& =\left(\sum_{k \geq 0}\left|h_{k}-h_{k+1}\right|^{n}\right)^{1 / n} \leq 1 .
\end{aligned}
$$

Moreover,

$$
\begin{aligned}
\int_{1}^{\infty} u^{n} t^{n-\beta-1} d t & =\sum_{k \geq 0} \int_{e^{\frac{k}{n}}}^{e^{\frac{k+1}{n}}} u^{n} t^{n-\beta-1} d t \\
& =\sum_{k \geq 0}\left(u\left(e^{\frac{k+1}{n}}\right)\right)^{n} \int_{e^{\frac{k}{n}}}^{e^{\frac{k+1}{n}}} t^{n-\beta-1} d t \\
& \gtrsim \sum_{k \geq 0}\left(u\left(e^{\frac{k+1}{n}}\right)\right)^{n} e^{\left(1-\frac{\beta}{n}\right)(k+1)} \\
& =\sum_{k \geq 1}\left(u\left(e^{\frac{k}{n}}\right)\right)^{n} e^{\left(1-\frac{\beta}{n}\right) k} \\
& \gtrsim \sum_{k \geq 1} h_{k}^{n} e^{\left(1-\frac{\beta}{n}\right) k} \\
& \gtrsim \sum_{k \geq 1} a_{k}^{n} e^{\left(1-\frac{\beta}{n}\right) k} .
\end{aligned}
$$


Therefore,

$$
\begin{aligned}
\|a\|_{(e)} & =a_{0}^{n}+\sum_{k \geq 1} a_{k}^{n} e^{\left(1-\frac{\beta}{n}\right) k} \\
& \leq h_{0}^{n}+\sum_{k \geq 1} a_{k}^{n} e^{\left(1-\frac{\beta}{n}\right) k} \\
& \lesssim h_{0}^{n}+\int_{1}^{\infty} u^{n} t^{n-\beta-1} d t .
\end{aligned}
$$

Next, we start to estimate $h_{0}$. Set $1<r<e^{1 / 4 n}$, then

$$
\begin{aligned}
h_{0}-\alpha_{n}^{\frac{n-1}{n}} u(r) & =\alpha_{n}^{\frac{n-1}{n}} \int_{1}^{r}\left|u^{\prime}(t)\right| d t \\
& =\alpha_{n}^{\frac{n-1}{n}}\left(\int_{1}^{r}\left|u^{\prime}(t)\right|^{n} t^{n-1} d t\right)^{\frac{1}{n}}\left(\int_{1}^{r} \frac{1}{t} d t\right)^{\frac{n-1}{n}} \\
& =4^{-\frac{n-1}{n}}\left(\omega_{n-1} \int_{1}^{r}\left|u^{\prime}(t)\right|^{n} t^{n-1} d t\right)^{\frac{1}{n}} \\
& \leq \frac{h_{0}}{2}
\end{aligned}
$$

and

$$
\int_{1}^{\infty} u^{n} t^{n-\beta-1} d t \geq \int_{1}^{e^{1 / 4 n}} u^{n} t^{n-\beta-1} d t \gtrsim h_{0}^{n}
$$

By (3) and (4), we derive that

$$
\|a\|_{(e)} \lesssim \int_{1}^{\infty} u^{n} t^{n-\beta-1} d t
$$

Then we apply Lemma 3 to conclude that

$$
\begin{aligned}
\int_{1}^{\infty} u^{n} t^{n-\beta-1} d t & \gtrsim \frac{e^{\left(1-\frac{\beta}{n}\right) h_{0}^{\frac{n}{n-1}}}}{h_{0}^{\frac{n}{n-1}}} \\
& =\frac{e^{\alpha_{n}\left(1-\frac{\beta}{n}\right) u^{\frac{n}{n-1}}(1)}}{\alpha_{n} u^{\frac{n}{n-1}(1)}} .
\end{aligned}
$$

This completes the proof of Lemma 4.

\section{Singular Moser-Trudinger inequality with the exact growth condition}

In this section, we shall establish a singular version of Moser-Trudinger inequality with the exact growth condition on hyperbolic space. Namely, we will give the proof of Theorem 1 . By the density, we can assume that $u(x)$ is compactly supported in $\mathbb{H}^{n}$. We use the idea of Moser [5]. Set $d(0, x)=t$ and $u(x)=v(d(0, x))=v(t)$, then

$$
\int_{\mathbb{H}^{n}} \frac{\Phi\left(\alpha_{n}\left(1-\frac{\beta}{n}\right)|u|^{\frac{n}{n-1}}\right)}{(1+u)^{\frac{n}{n-1}}(d(0, x))^{\beta}} d V=\omega_{n-1} \int_{0}^{\infty} \frac{\Phi\left(\alpha_{n}\left(1-\frac{\beta}{n}\right)|v|^{\frac{n}{n-1}}\right)}{(1+v)^{\frac{n}{n-1}}} t^{-\beta}(\sinh t)^{n-1} d t,
$$




$$
\begin{aligned}
& \int_{\mathbb{H}^{n}}\left|\nabla_{g} u(x)\right|^{n} d V=\omega_{n-1} \int_{0}^{\infty}\left|v^{\prime}(t)\right|^{n}(\sinh t)^{n-1} d t, \\
& \int_{\mathbb{H}^{n}} \frac{|u(x)|^{n}}{(d(0, x))^{\beta}} d V=\omega_{n-1} \int_{0}^{\infty}|v(t)|^{n}(\sinh t)^{n-1} t^{-\beta} d t .
\end{aligned}
$$

Thus, it suffices to show that there exists a positive constant $C_{n}$ such that

$$
\int_{0}^{\infty} \frac{\Phi\left(\alpha_{n}\left(1-\frac{\beta}{n}\right)|v|^{\frac{n}{n-1}}\right)}{(1+v)^{\frac{n}{n-1}}} t^{-\beta}(\sinh t)^{n-1} d t \leq C_{n} \int_{0}^{\infty}|v(t)|^{n}(\sinh t)^{n-1} t^{-\beta} d t
$$

for any $v(t)$ satisfying $v(t) \geq 0, v^{\prime}(t) \leq 0, v\left(t_{0}\right)=0$ for some $t_{0} \in \mathbb{R}$ and

$$
\omega_{n-1} \int_{0}^{\infty}\left|v^{\prime}(t)\right|^{n}(\sinh t)^{n-1} d t \leq 1 .
$$

Set $R=\sup \{t \in \mathbb{R}: v(t) \geq 1\}$. We can use $v(t) \in(0,1)$ for $t \in(R, \infty)$ to obtain

$$
\Phi\left(\alpha_{n}\left(1-\frac{\beta}{n}\right)|v|^{\frac{n}{n-1}}\right) \leq C_{n}|v(t)|^{n}
$$

for any $t \geq R$. It follows that

$$
\begin{aligned}
& \int_{R}^{\infty} \frac{\Phi\left(\alpha_{n}\left(1-\frac{\beta}{n}\right)|v|^{\frac{n}{n-1}}\right)}{(1+v)^{\frac{n}{n-1}}} t^{-\beta}(\sinh t)^{n-1} d t \\
& \quad \leq \int_{R}^{\infty} \Phi\left(\alpha_{n}\left(1-\frac{\beta}{n}\right)|v|^{\frac{n}{n-1}}\right) t^{-\beta}(\sinh t)^{n-1} d t \\
& \quad \leq C_{n} \int_{R}^{\infty}|v(t)|^{n} t^{-\beta}(\sinh t)^{n-1} d t .
\end{aligned}
$$

Next, we focus on the integral over $(0, R]$. Set $0<\varepsilon_{0}<1$ and let $R_{1}(u)>0$ such that

$$
\omega_{n-1} \int_{0}^{R_{1}}|v(t)|^{n}(\sinh t)^{n-1} d t \leq \rho \varepsilon_{0}
$$

and

$$
\omega_{n-1} \int_{R_{1}}^{\infty}|v(t)|^{n}(\sinh t)^{n-1} d t \leq \rho\left(1-\varepsilon_{0}\right),
$$

where $0<\rho \leq 1$.

In order to estimate the integral over $(0, R]$, we need to consider two cases: $R_{1} \geq R$ and $R_{1} \leq R$.

First, we consider the case that $R_{1} \geq R$. For $0<t \leq R$, we can write

$$
\begin{aligned}
v(t) & =v(R)+\int_{R}^{t} v^{\prime}(s) d s \\
& \leq v(R)+\left(\int_{t}^{R}\left|v^{\prime}(s)\right|^{n}(\sinh s)^{n-1} d s\right)^{\frac{1}{n}}\left(\int_{t}^{R} \frac{1}{\sinh s} d s\right)^{\frac{n-1}{n}} \\
& \leq 1+\left(\frac{\rho \varepsilon_{0}}{w_{n-1}}\right)^{\frac{1}{n}}\left(\ln \left(\frac{e^{R}-1}{e^{R}+1} \frac{e^{t}+1}{e^{t}-1}\right)\right)^{\frac{n-1}{n}} .
\end{aligned}
$$


Lu and Chen Journal of Inequalities and Applications <wide>(2017<wide>) 2017:127

Page 8 of 14

For any $\varepsilon>0$, one can apply the following well-known inequality:

$$
1+s^{\frac{n-1}{n}} \leq\left((1+\varepsilon) s+C_{\varepsilon}\right)^{\frac{n-1}{n}}
$$

to obtain

$$
|v(t)|^{\frac{n}{n-1}} \leq(1+\varepsilon)\left(\frac{\rho \varepsilon_{0}}{w_{n-1}}\right)^{\frac{1}{n-1}}\left(\ln \left(\frac{e^{R}-1}{e^{R}+1} \frac{e^{t}+1}{e^{t}-1}\right)\right)+C_{\varepsilon}
$$

Pick $\varepsilon$ sufficiently small such that $(1+\varepsilon)^{n-1} \varepsilon_{0}<1$, then

$$
|v(t)|^{\frac{n}{n-1}} \leq\left(\frac{\rho(1+\varepsilon)^{n-1} \varepsilon_{0}}{w_{n-1}}\right)^{\frac{1}{n-1}}\left(\ln \left(\frac{e^{R}-1}{e^{R}+1} \frac{e^{t}+1}{e^{t}-1}\right)\right)+C_{\varepsilon_{0}} .
$$

Denote $c_{0}=(n-\beta)\left(\rho(1+\varepsilon)^{n-1} \varepsilon_{0}\right)^{\frac{1}{n-1}}$. It follows that $0<c_{0}<n-\beta$ and

$$
\begin{aligned}
\int_{0}^{R} & \frac{\Phi\left(\alpha_{n}\left(1-\frac{\beta}{n}\right)|v|^{\frac{n}{n-1}}\right)}{(1+v)^{\frac{n}{n-1}}} t^{-\beta}(\sinh t)^{n-1} d t \\
\leq & \int_{0}^{R} \Phi\left(\alpha_{n}\left(1-\frac{\beta}{n}\right)|v|^{\frac{n}{n-1}}\right) t^{-\beta}(\sinh t)^{n-1} d t \\
\leq & \int_{0}^{R} \Phi\left(\alpha_{n}\left(1-\frac{\beta}{n}\right)\left(\left(\frac{\rho(1+\varepsilon)^{n-1} \varepsilon_{0}}{w_{n-1}}\right)^{\frac{1}{n-1}} \ln \left(\frac{e^{R}-1}{e^{R}+1} \frac{e^{t}+1}{e^{t}-1}\right)+C_{\varepsilon_{0}}\right)\right) \\
& \times t^{-\beta}(\sinh t)^{n-1} d t \\
\leq & \exp \left(\alpha_{n}\left(1-\frac{\beta}{n}\right) C_{\varepsilon_{0}}\right)\left(\frac{e^{R}-1}{e^{R}+1}\right)^{c_{0}} \int_{0}^{R} \frac{\left(e^{t}+1\right)^{c_{0}+n-1}}{\left(e^{t}-1\right)^{c_{0}-n+1}} \frac{t^{-\beta}}{\left(2 e^{t}\right)^{n-1}} d t .
\end{aligned}
$$

For $R \rightarrow 0$, it is easy to check that

$$
\exp \left(\alpha_{n}\left(1-\frac{\beta}{n}\right) C_{\varepsilon_{0}}\right)\left(\frac{e^{R}-1}{e^{R}+1}\right)^{c_{0}} \int_{0}^{R} \frac{\left(e^{t}+1\right)^{c_{0}+n-1}}{\left(e^{t}-1\right)^{c_{0}-n+1}} \frac{t^{-\beta}}{\left(2 e^{t}\right)^{n-1}} d t \sim R^{n-\beta} .
$$

For $R \rightarrow+\infty$, one can calculate

$$
\begin{aligned}
& \exp \left(\alpha_{n}\left(1-\frac{\beta}{n}\right) C_{\varepsilon_{0}}\right)\left(\frac{e^{R}-1}{e^{R}+1}\right)^{c_{0}} \int_{0}^{R} \frac{\left(e^{t}+1\right)^{c_{0}+n-1}}{\left(e^{t}-1\right)^{c_{0}-n+1}} \frac{t^{-\beta}}{\left(2 e^{t}\right)^{n-1}} d t \\
& \sim \int_{0}^{R} \frac{\left(e^{t}+1\right)^{c_{0}+n-1}}{\left(e^{t}-1\right)^{c_{0}-n+1}} \frac{t^{-\beta}}{\left(2 e^{t}\right)^{n-1}} d t .
\end{aligned}
$$

On the other hand,

$$
\int_{0}^{R}|v(t)|^{n}(\sinh t)^{n-1} t^{-\beta} d t \geq \int_{0}^{R}(\sinh t)^{n-1} t^{-\beta} d t
$$

Therefore, for $R \rightarrow 0$,

$$
\int_{0}^{R}(\sinh t)^{n-1} t^{-\beta} d t \sim R^{n-\beta}
$$


Lu and Chen Journal of Inequalities and Applications <wide>(2017<wide>) 2017:127

Page 9 of 14

Thus,

$$
\begin{aligned}
& \lim _{R \rightarrow 0} \frac{\int_{0}^{R}|v(t)|^{n}(\sinh t)^{n-1} t^{-\beta} d t}{\int_{0}^{R} \frac{\Phi\left(\alpha_{n}\left(1-\frac{\beta}{n}\right)|v| \frac{n}{n-1}\right)}{(1+v)^{n-1}} t^{-\beta}(\sinh t)^{n-1} d t} \\
& \quad \geq \lim _{R \rightarrow 0} \frac{\int_{0}^{R}(\sinh t)^{n-1} t^{-\beta} d t}{\exp \left(\alpha_{n}\left(1-\frac{\beta}{n}\right) C_{\varepsilon_{0}}\right)\left(\frac{e^{R}-1}{e^{R}+1}\right)^{c_{0}} \int_{0}^{R} \frac{\left(e^{t}+1\right)^{c_{0}+n-1}}{\left(e^{t}-1\right)^{c^{-n+1}}} \frac{t^{-\beta}}{\left(2 e^{t}\right)^{n-1}} d t} \\
& \quad \geq C_{0}
\end{aligned}
$$

and

$$
\begin{aligned}
& \lim _{R \rightarrow+\infty} \frac{\int_{0}^{R}|v(t)|^{n}(\sinh t)^{n-1} t^{-\beta} d t}{\int_{0}^{R} \frac{\Phi\left(\alpha_{n}\left(1-\frac{\beta}{n}\right)|v|^{\frac{n}{n-1}}\right)}{(1+v)^{n-1}} t^{-\beta}(\sinh t)^{n-1} d t} \\
& \quad \geq \lim _{R \rightarrow+\infty} \frac{\int_{0}^{R}(\sinh t)^{n-1} t^{-\beta} d t}{\exp \left(\alpha_{n}\left(1-\frac{\beta}{n}\right) C_{\varepsilon_{0}}\right)\left(\frac{e^{R}-1}{e^{R}+1}\right)^{c_{0}} \int_{0}^{R} \frac{\left(e^{t}+1\right)^{c_{0}+n-1}}{\left(e^{t}-1\right)^{c_{0}-n+1}} \frac{t^{-\beta}}{\left(2 e^{t}\right)^{n-1}} d t} \\
& \geq \lim _{R \rightarrow+\infty} \frac{(\sinh R)^{n-1} R^{-\beta}}{\exp \left(\alpha_{n}\left(1-\frac{\beta}{n}\right) C_{\varepsilon_{0}}\right) R^{-\beta}\left(e^{R}\right)^{2 n-2}\left(2 e^{R}\right)^{1-n}} \\
& \quad=C_{1} .
\end{aligned}
$$

We can combine (7) and (8) to derive that

$$
\begin{gathered}
\int_{0}^{R} \frac{\Phi\left(\alpha_{n}\left(1-\frac{\beta}{n}\right)|v|^{\frac{n}{n-1}}\right)}{(1+v)^{\frac{n}{n-1}}} t^{-\beta}(\sinh t)^{n-1} d t \\
\leq C_{n} \int_{0}^{R}|v(t)|^{n}(\sinh t)^{n-1} t^{-\beta} d t .
\end{gathered}
$$

Then by (5) and (9), we obtain the desired inequality of Theorem 1 for $R_{1} \geq R$.

Now, we consider the case $R_{1}<R$. First, we consider the integral over $\left(R_{1}, R\right)$. By $\omega_{n-1} \int_{R_{1}}^{\infty}|v(t)|^{n}(\sinh t)^{n-1} d t \leq \rho \varepsilon_{0}$, we have

$$
\begin{aligned}
v(t) & =v(R)+\int_{R}^{t} v^{\prime}(s) d s \\
& \leq v(R)+\left(\int_{R_{1}}^{\infty}\left|v^{\prime}(s)\right|^{n}(\sinh s)^{n-1} d s\right)^{\frac{1}{n}}\left(\int_{t}^{R} \frac{1}{\sinh s} d s\right)^{\frac{n-1}{n}} \\
& \leq 1+\left(\frac{\rho \varepsilon_{0}}{w_{n-1}}\right)^{\frac{1}{n}}\left(\ln \left(\frac{e^{R}-1}{e^{R}+1} \frac{e^{t}+1}{e^{t}-1}\right)\right)^{\frac{n-1}{n}}
\end{aligned}
$$

where $R_{1}<t<R$. Set $\varepsilon=\left(1+\varepsilon_{0}\right)^{\frac{1}{n-1}}-1$. It is easy to check that

$$
|v(t)|^{\frac{n}{n-1}} \leq\left(\frac{\rho\left(1-\varepsilon_{0}^{2}\right)}{w_{n-1}}\right)^{\frac{1}{n-1}}\left(\ln \left(\frac{e^{R}-1}{e^{R}+1} \frac{e^{t}+1}{e^{t}-1}\right)\right)+C_{\varepsilon_{0}}
$$


by (6). Denote $c_{1}=(n-\beta)\left(\rho\left(1-\varepsilon_{0}^{2}\right)\right)^{\frac{1}{n-1}}$, then $0<c_{1}<n-\beta$ and

$$
\begin{aligned}
& \int_{R_{1}}^{R} \frac{\Phi\left(\alpha_{n}\left(1-\frac{\beta}{n}\right)|v| \frac{n}{n-1}\right)}{(1+v)^{\frac{n}{n-1}}} t^{-\beta}(\sinh t)^{n-1} d t \\
& \quad \leq \int_{R_{1}}^{R} \Phi\left(\alpha_{n}\left(1-\frac{\beta}{n}\right)|v| \frac{n}{n-1}\right) t^{-\beta}(\sinh t)^{n-1} d t \\
& \quad \leq \int_{0}^{R} \Phi\left(\alpha_{n}\left(1-\frac{\beta}{n}\right)\left(\frac{\rho\left(1-\varepsilon_{0}^{2}\right)}{w_{n-1}}\right)^{\frac{1}{n-1}}\left(\ln \left(\frac{e^{R}-1}{e^{R}+1} \frac{e^{t}+1}{e^{t}-1}\right)\right)+C_{\varepsilon_{0}}\right) t^{-\beta}(\sinh t)^{n-1} d t \\
& \quad \leq \exp \left(\alpha_{n}\left(1-\frac{\beta}{n}\right) C_{\varepsilon_{0}}\right)\left(\frac{e^{R}-1}{e^{R}+1}\right)^{c_{1}} \int_{0}^{R} \frac{\left(e^{t}+1\right)^{c_{1}+n-1}}{\left(e^{t}-1\right)^{c_{1}-n+1}} \frac{t^{-\beta}}{\left(2 e^{t}\right)^{n-1}} d t .
\end{aligned}
$$

Using the same calculation as we did in the case $R_{1} \geq R$, we can derive

$$
\int_{R_{1}}^{R} \frac{\Phi\left(\alpha_{n}\left(1-\frac{\beta}{n}\right)|v|^{\frac{n}{n-1}}\right)}{(1+v)^{\frac{n}{n-1}}} t^{-\beta}(\sinh t)^{n-1} d t \leq C_{n} \int_{0}^{R}|v(t)|^{n}(\sinh t)^{n-1} t^{-\beta} d t
$$

Now, we only need to consider the integral on $\left[0, R_{1}\right)$. Set $w(t)=v(t)-v\left(R_{1}\right)$, then

$$
\omega_{n-1} \int_{0}^{R_{1}}\left|w^{\prime}(t)\right|^{n}(\sinh t)^{n-1} d t \leq \rho \varepsilon_{0} .
$$

One can employ the equality (6) to derive that

$$
|v(t)|^{\frac{n}{n-1}}=(1+\varepsilon) w(t)^{\frac{n}{n-1}}+C_{\varepsilon} v\left(R_{1}\right)^{\frac{n}{n-1}} .
$$

Then we obtain

$$
\begin{aligned}
\int_{0}^{R_{1}} & \frac{\Phi\left(\alpha_{n}\left(1-\frac{\beta}{n}\right)|v|^{\frac{n}{n-1}}\right)}{(1+v)^{\frac{n}{n-1}}} t^{-\beta}(\sinh t)^{n-1} d t \\
\leq & \int_{0}^{R_{1}} \frac{\Phi\left(\alpha_{n}\left(1-\frac{\beta}{n}\right)|v|^{\frac{n}{n-1}}\right)}{\left(v\left(R_{1}\right)\right)^{\frac{n}{n-1}}} t^{-\beta}(\sinh t)^{n-1} d t \\
\leq & \frac{\exp \left(\alpha_{n}\left(1-\frac{\beta}{n}\right) C_{\varepsilon} v\left(R_{1}\right)^{\frac{n}{n-1}}\right)}{\left(v\left(R_{1}\right)\right)^{\frac{n}{n-1}}} \\
& \times \int_{0}^{R_{1}} \exp \left(\alpha_{n}\left(1-\frac{\beta}{n}\right)(1+\varepsilon) w^{\frac{n}{n-1}}\right) t^{-\beta}(\sinh t)^{n-1} d t .
\end{aligned}
$$

Set $\varepsilon=\varepsilon_{0}^{\frac{1}{1-n}}-1$, then

$$
C_{\varepsilon}=\left(1-\frac{1}{(1+\varepsilon)^{n-1}}\right)^{\frac{1}{1-n}}=\left(1-\varepsilon_{0}\right)^{\frac{1}{1-n}} .
$$

Since

$$
\omega_{n-1} \int_{R_{1}}^{\infty}\left|v^{\prime}(t)\right|^{n}(\sinh t)^{n-1} d t \leq \rho\left(1-\varepsilon_{0}\right) \leq 1-\varepsilon_{0} .
$$


We can apply Lemma 4 to obtain

$$
\frac{\exp \left(\alpha_{n}\left(1-\frac{\beta}{n}\right) C_{\varepsilon} v\left(R_{1}\right)^{\frac{n}{n-1}}\right)}{\left(v\left(R_{1}\right)\right)^{\frac{n}{n-1}}} \leq C_{\varepsilon_{0}} \frac{\int_{R_{1}}^{\infty}|v|^{n} t^{n-1} d t}{\left(1-\varepsilon_{0}\right)^{\frac{n}{n-1}}\left(R_{1}\right)^{n-\beta}} .
$$

Let $\Omega=\left\{x: d(0, x)<R_{1}\right\}$ and $v_{1}(x)=(1+\varepsilon)^{\frac{n-1}{n}} w(d(0, x))$ in $\Omega$, then

$$
\int_{\mathbb{H}^{n}}\left|\nabla_{g} v_{1}\right|^{n} d V=\omega_{n-1} \int_{0}^{R_{1}}(1+\varepsilon)^{n-1}\left|w^{\prime}(t)\right|^{n}(\sinh t)^{n-1} d t \leq \rho \leq 1 .
$$

We can apply the singular Moser-Trudinger inequality on a bounded domain to obtain

$$
\int_{\Omega} \exp \left(\alpha_{n}\left(1-\frac{\beta}{n}\right)\left|v_{1}\right|^{\frac{n}{n-1}}\right)(d(0, x))^{-\beta} d V \leq C \int_{\Omega}(d(0, x))^{-\beta} d V
$$

That is,

$$
\int_{0}^{R_{1}} \exp \left(\alpha_{n}\left(1-\frac{\beta}{n}\right)(1+\varepsilon)|w|^{\frac{n}{n-1}}\right) t^{-\beta}(\sinh t)^{n-1} d t \leq C \int_{0}^{R_{1}} t^{-\beta}(\sinh t)^{n-1} d t
$$

Since $\frac{\sinh t}{t}$ is monotone increasing on $(0, \infty)$, by (11), (12) and (13) we derive

$$
\begin{aligned}
& \int_{0}^{R_{1}} \frac{\Phi\left(\alpha_{n}\left(1-\frac{\beta}{n}\right)|v|^{\frac{n}{n-1}}\right)}{(1+v)^{\frac{n}{n-1}}} t^{-\beta}(\sinh t)^{n-1} d t \\
& \quad \leq C_{\varepsilon_{0}} \frac{\int_{R_{1}}^{\infty}|v|^{n} t^{n-1-\beta} d t}{\left(R_{1}\right)^{n-\beta}} \int_{0}^{R_{1}} t^{-\beta}(\sinh t)^{n-1} d t \\
& \quad \leq C_{\varepsilon_{0}} \frac{\int_{R_{1}}^{\infty}|v|^{n}(\sinh t)^{n-1}\left(\frac{t}{\sinh t}\right)^{n-1} t^{-\beta} d t}{\left(R_{1}\right)^{n-\beta}} \int_{0}^{R_{1}} t^{n-1-\beta}\left(\frac{\sinh t}{t}\right)^{n-1} d t \\
& \quad \leq C_{\varepsilon_{0}} \int_{R_{1}}^{\infty}|v|^{n}(\sinh t)^{n-1} t^{-\beta} d t .
\end{aligned}
$$

Combining (10) with (14), we obtain the desired inequality of Theorem 1 for $R_{1} \leq R$. This accomplishes the proof of Theorem 1 .

\section{Sharpness}

In this section, we show that the desired inequality in Theorem 1 does not hold if the power $\frac{n}{n-1}$ is replaced by any $p<\frac{n}{n-1}$.

We choose $\left\{u_{k}\right\}_{k=1}^{\infty}$ as follows:

$$
u_{k}(x)=\omega_{n-1}^{-\frac{1}{n}} C_{k} \begin{cases}k^{\frac{n-1}{n}}, & \text { if } 0 \leq d(0, x) \leq e^{-k} \\ |\ln d(0, x)| k^{-\frac{1}{n}}, & \text { if } e^{-k}<d(0, x) \leq 1 \\ 0, & \text { if } d(0, x)>1\end{cases}
$$


where $C_{k}=\left(k^{-1} \int_{e^{-k}}^{1} t^{-n}(\sinh t)^{n-1} d t\right)^{-\frac{1}{n}}$. It is easy to check that $C_{k} \rightarrow 1$ and $C_{k}^{\frac{n}{n-1}} k-k \rightarrow 0$ as $k \rightarrow \infty$. Let $v_{k}(d(0, x))=u_{k}(x)$, then

$$
v_{k}(t)=\omega_{n-1}^{-\frac{1}{n}} C_{k} \begin{cases}k^{\frac{n-1}{n}}, & \text { if } 0 \leq t \leq e^{-k} \\ |\ln t| k^{-\frac{1}{n}}, & \text { if } e^{-k}<t \leq 1 \\ 0, & \text { if } t>1 .\end{cases}
$$

By calculation, we derive that

$$
\begin{aligned}
\int_{\mathbb{H}^{n}}\left|\nabla_{g} u_{k}\right|^{n} d V & =\omega_{n-1} \int_{0}^{\infty}\left|v_{k}(t)\right|^{n}(\sinh t)^{n-1} d t \\
& =\frac{\left(C_{k}\right)^{n}}{k} \int_{e^{-k}}^{1} t^{-n}(\sinh t)^{n-1} d t \\
& =1
\end{aligned}
$$

and

$$
\begin{aligned}
\int_{\mathbb{H}^{n}} & \frac{\left|u_{k}(x)\right|^{n}}{(d(0, x))^{\beta}} d V \\
= & \omega_{n-1} \int_{0}^{\infty}\left|v_{k}(t)\right|^{n}(\sinh t)^{n-1} t^{-\beta} d t \\
= & \left(C_{k}\right)^{n} \int_{0}^{e^{-k}} k^{n-1}(\sinh t)^{n-1} t^{-\beta} d t \\
& +\left(C_{k}\right)^{n} \int_{e^{-k}}^{1} k^{-1}|\ln t|^{n}(\sinh t)^{n-1} t^{-\beta} d t \\
= & O\left(\frac{k^{n-1}}{e^{(n-\beta) k}}\right)+O\left(\frac{1}{k}\right) \\
= & O\left(\frac{1}{k}\right) .
\end{aligned}
$$

It follows that

$$
\begin{aligned}
& \int_{\mathbb{H}^{n}} \frac{\Phi\left(\alpha_{n}\left(1-\frac{\beta}{n}\right)\left|u_{k}\right|^{\frac{n}{n-1}}\right)}{\left(1+u_{k}\right)^{p}(d(0, x))^{\beta}} d V \\
&= \omega_{n-1} \int_{0}^{\infty} \frac{\Phi\left(\alpha_{n}\left(1-\frac{\beta}{n}\right)\left|v_{k}\right|^{\frac{n}{n-1}}\right)}{\left(1+v_{k}\right)^{p}} t^{-\beta}(\sinh t)^{n-1} d t \\
& \geq \frac{\Phi\left(\alpha_{n}\left(1-\frac{\beta}{n}\right)\left|\omega_{n-1}^{-\frac{1}{n}} C_{k} k^{\frac{n-1}{n}}\right| \frac{n}{n-1}\right)}{\left(1+\left|\omega_{n-1}^{-\frac{1}{n}} C_{k} k^{\frac{n-1}{n}}\right|\right)^{p}} \int_{0}^{e^{-k}}(\sinh t)^{n-1} t^{-\beta} d t \\
& \sim \frac{\Phi\left((n-\beta) k C_{k}^{\frac{n}{n-1}}\right)}{\left(1+\left|\omega_{n-1}^{-\frac{1}{n}} C_{k} k^{\frac{n-1}{n}}\right|\right)^{p}} e^{-(n-\beta) k} \\
& \sim k^{-\frac{n-1}{n}}
\end{aligned}
$$

as $k \rightarrow \infty$. 
When $p<\frac{n}{n-1}$, we can apply (15) and (16) to obtain

$$
\begin{aligned}
& \int_{\mathbb{H}^{n}} \frac{\Phi\left(\alpha_{n}\left(1-\frac{\beta}{n}\right)\left|u_{k}\right|^{\frac{n}{n-1}}\right)}{\left(1+u_{k}\right)^{p}(d(0, x))^{\beta}} d V\left(\int_{\mathbb{H}^{n}} \frac{\left|u_{k}(x)\right|^{n}}{(d(0, x))^{\alpha}} d V\right)^{-1} \\
& \quad \geq k^{1-p \frac{n-1}{n}} \\
& \quad \rightarrow \infty
\end{aligned}
$$

Thus, we accomplish the proof of Theorem 2.

\section{Conclusions}

In this paper, we prove a singular version of Moser-Trudinger inequality with the exact growth condition in the $n$-dimension hyperbolic space $\mathbb{H}^{n}$. It is well known that the MoserTrudinger inequality plays an important role in nonlinear analysis and can be applied to study the ground state solutions of $N$-Laplacian equation with critical exponential growth. Our results represent very good progress on modern analysis and geometric inequalities.

Acknowledgements

The authors would like to thank the anonymous referee for his/her valuable comments. The work is partly supported by the Scientific Research Fund of Jiangxi Provincial Education Department (No. GJJ160797).

\section{Competing interests}

The authors declare that they have no competing interests.

\section{Authors' contributions}

All authors conceived of the study, participated in its design and coordination, drafted the manuscript, participated in the sequence alignment. All authors read and approved the final manuscript.

\section{Author details}

'School of Mathematics and Computer Science, Jiangxi Science and Technology Normal University, Nanchang, 330038,

China. ${ }^{2}$ School of Mathematical Sciences, Beijing Normal University, Beijing, 100875, China.

\section{Publisher's Note}

Springer Nature remains neutral with regard to jurisdictional claims in published maps and institutional affiliations.

Received: 12 November 2016 Accepted: 17 May 2017 Published online: 02 June 2017

\section{References}

1. Lu, G, Tang, H: Sharp Moser-Trudinger inequalities on hyperbolic spaces with exact growth condition. J. Geom. Anal. 26, 837-857 (2016)

2. Yudovic, Vl: Some estimates connected with integral operators and with solutions of elliptic equations. Dokl. Akad. Nauk SSSR 138, 805-808 (1961) (Russian)

3. Pohozaev, SI: On the eigenfunctions of the equation $\Delta u+\lambda f(u)=0$. Dokl. Akad. Nauk SSSR 165, 36-39 (1965) (Russian)

4. Trudinger, NS: On embeddings into Orlicz spaces and some applications. J. Math. Mech. 17, 473-483 (1967)

5. Moser, J: A sharp form of an inequality by N. Trudinger. Indiana Univ. Math. J. 20, 1077-1092 (1970)

6. Carleson, L, Chang, SYA: On the existence of an extremal function for an inequality of J. Moser. Bull. Sci. Math. 110, 113-127 (1986)

7. Flucher, M: Extremal functions for Trudinger-Moser inequality in 2 dimensions. Comment. Math. Helv. 67, 471-497 (1992)

8. Lin, K: Extremal functions for Moser's inequality. Trans. Am. Math. Soc. 348, 2663-2671 (1996)

9. Li, YX: Moser-Trudinger inequality on compact Riemannian manifolds of dimension two. J. Partial Differ. Equ. 14 163-192 (2001)

10. Li, YX: Extremal functions for the Moser-Trudinger inequalities on compact Riemannian manifolds. Sci. China Ser. A 48, 618-648 (2005)

11. Cohn, WS, Lu, G: Best constants for Moser-Trudinger inequalities on the Heisenberg group. Indiana Univ. Math. J. 50, 1567-1591 (2001)

12. Lam, N, Lu, G, Tang, H: Sharp subcritical Moser-Trudinger inequalities on Heisenberg groups and subelliptic PDEs. Nonlinear Anal. 95, 77-92 (2014)

13. Adimurthi, A, Sandeep, K: A singular Moser-Trudinger embedding and its applications. Nonlinear Differ. Equ. Appl. 13, 585-603 (2007) 
14. Adimurthi, A, Yang, Y: An interpolation of Hardy inequality and Trundinger-Moser inequality in $\mathbb{R}^{N}$ and its applications. Int. Math. Res. Not. 13, 2394-2426 (2010)

15. Chang, SYA, Yang, P: The inequality of Moser and Trudinger and applications to conformal geometry. Dedicated to the memory of Jürgen K. Moser. Commun. Pure Appl. Math. 56, 1135-1150 (2003)

16. Cohn, WS, Lu, G: Sharp constants for Moser-Trudinger inequalities on spheres in complex space $C^{n}$. Commun. Pure Appl. Math. 57, 1458-1493 (2004)

17. Dong, M, Lu, G: Best constants and existence of maximizers for weighted Trudinger-Moser inequalities. Calc. Var. Partial Differ. Equ. 55, 55-88 (2016)

18. de Souza, M, do Ó, JM: On singular Trudinger-Moser type inequalities for unbounded domains and their best exponents. Potential Anal. 38, 1091-1101 (2013)

19. Lam, N, Lu, G, Tang, H: On nonuniformly subelliptic equations of Q-sub-Laplacian type with critical growth in $H^{n}$. Adv. Nonlinear Stud. 12, 659-681 (2012)

20. Adachi, S, Tanaka, K: Trudinger type inequalities in $\mathbb{R}^{N}$ and their best exponents. Proc. Am. Math. Soc. 128, 2051-2057 (1999)

21. Ruf, B: A sharp Moser-Trudinger type inequality for unbounded domains in $R^{2}$. J. Funct. Anal. 219, 340-367 (2005)

22. Li, YX, Ruf, B: A sharp Moser-Trudinger type inequality for unbounded domains in $\mathbb{R}^{n}$. Indiana Univ. Math. J. 57, 451-480 (2008)

23. Masmoudi, N, Sani, F: Adams' inequality with the exact growth condition in $\mathbb{R}^{4}$. Commun. Pure Appl. Math. 67, 1307-1335 (2014)

24. Masmoudi, N, Sani, F: Trudinger-Moser inequalities with the exact growth condition in $\mathbb{R}^{n}$ and application. Commun. Partial Differ. Equ. 40, 1408-1440 (2015)

25. Chen, L, Liu, Z: Singular Moser-Trudinger inequality with the exact growth condition in $\mathbb{R}^{n}$. Math. Inequal. Appl. 20, 845-854 (2017)

26. Mancini, G, Sandeep, K: Moser-Trudinger inequalities on conformal discs. Commun. Contemp. Math. 12, 1055-1068 (2010)

27. Lu, G, Tang, H: Best constants for Moser-Trudinger inequalities on high dimensional hyperbolic spaces. Adv. Nonlinear Stud. 13, 1035-1052 (2013)

\section{Submit your manuscript to a SpringerOpen ${ }^{\mathcal{O}}$ journal and benefit from:}

- Convenient online submission

- Rigorous peer review

- Immediate publication on acceptance

- Open access: articles freely available online

- High visibility within the field

- Retaining the copyright to your article 